\title{
8
}

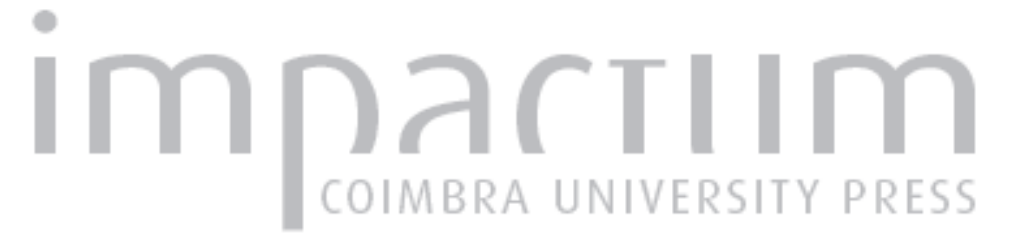

\section{Futurismo avanguardia italiana}

\section{Autor(es): Margozzi, Mariastella}

Publicado por: Imprensa da Universidade de Coimbra

URL persistente:

URl:http://hdl.handle.net/10316.2/42570

DOI:

DOI:https://doi.org/10.14195/0870-8584_4_1

Accessed : $\quad$ 26-Apr-2023 13:58:21

A navegação consulta e descarregamento dos títulos inseridos nas Bibliotecas Digitais UC Digitalis, UC Pombalina e UC Impactum, pressupõem a aceitação plena e sem reservas dos Termos e Condições de Uso destas Bibliotecas Digitais, disponíveis em https://digitalis.uc.pt/pt-pt/termos.

Conforme exposto nos referidos Termos e Condições de Uso, o descarregamento de títulos de acesso restrito requer uma licença válida de autorização devendo o utilizador aceder ao(s) documento(s) a partir de um endereço de IP da instituição detentora da supramencionada licença.

Ao utilizador é apenas permitido o descarregamento para uso pessoal, pelo que o emprego do(s) título(s) descarregado(s) para outro fim, designadamente comercial, carece de autorização do respetivo autor ou editor da obra.

Na medida em que todas as obras da UC Digitalis se encontram protegidas pelo Código do Direito de Autor e Direitos Conexos e demais legislação aplicável, toda a cópia, parcial ou total, deste documento, nos casos em que é legalmente admitida, deverá conter ou fazer-se acompanhar por este aviso.

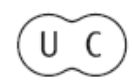




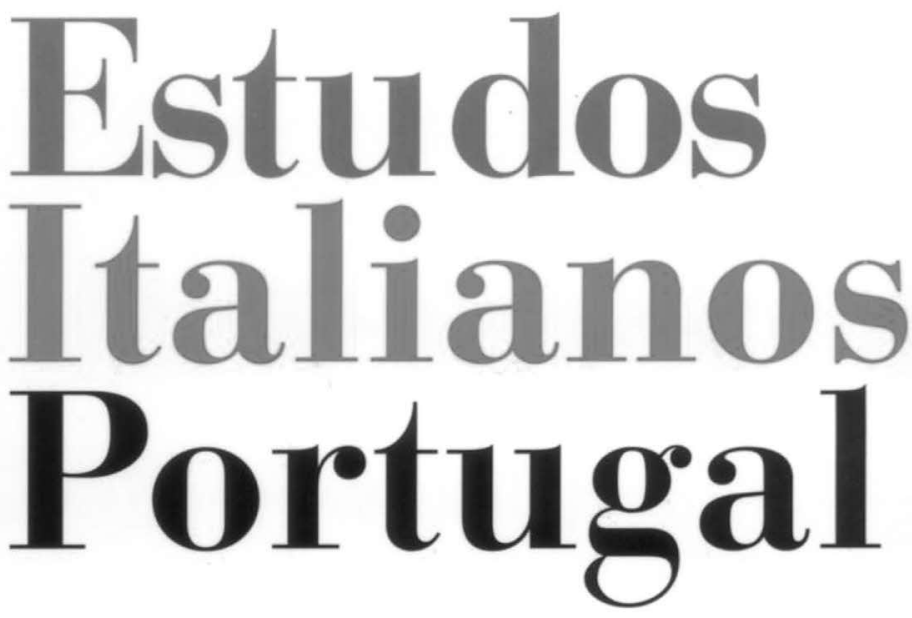

Instituto

Italiano

de Cultura

de Lisboa

Nova Série

$\mathbf{N}^{\mathbf{0}} 4$.

2009 


\title{
FUTURISMO AVANGUARDIA ITALIANA
}

\author{
Mariastella Margozzi
}

Il Futurismo, unico vero movimento di rinnovamento nell'Italia dell'inizio del ventesimo secolo, si identifica con il suo ideatore, il letterato Filippo Tommaso Marinetti, che il 20 febbraio 1909 ne pubblica il Manifesto su Le Figaro di Parigi, dando vita così non solo al movimento ma anche alla sua promozione e divulgazione attraverso la stampa e attraverso un mezzo, quello del "manifesto" appunto, che si rivelerà parte integrante della sua fortuna. Infatti, grazie a questo appello rivoluzionario, da quel momento molti degli artisti più giovani si associano alle sue idee, sia singolarmente che in gruppo, facendo registrare in tutt'Italia un fermento culturale innovativo mai avuto prima. Tra i primi ad aderire al movimento ci sono Carrà, Boccioni, Russolo, Severini, Bonzagni e Romani; tutti loro nel 1910 firmano insieme

* Laureata in Storia dell'Arte Moderna presso l'Università degli Studi La Sapienza di Roma e specializzata in Storia dell'Arte Medioevale e Moderna presso la stessa Università. Ha prestato servizio presso la Soprintendenza di Caserta e Benevento, Palazzo Reale di Caserta, la Galleria Nazionale d'Arte Moderna e Contemporanea di Roma, del Laboratorio di Restauro, e del Museo Boncompagni Ludovisi per le Arti decorative, il Costume e la Moda. Dal 1993 è anche responsabile del Settore Arte del Primo Novecento della stessa Galleria Nazionale. Ha svolto studi e approfondimenti su artisti e argomenti di arte moderna della prima metà del secolo XX. È curatrice della mostra Collezionare il Futurismo (Lisbona, Museu da Água, 16 dicembre 2009-31 gennaio 2010). 
a Marinetti il Manifesto dei pittori futuristi, mentre solo Boccioni, Carrà, Russolo, e Severini sottoscrivono il Manifesto tecnico della pittura futurista.

Nel 1914 sarà la volta di Antonio Sant'Elia a scrivere il Manifesto dell'architettura, ma altri manifesti saranno redatti e pubblicati negli anni a seguire. Il rinnovamento totale che il futurismo intende si oppone prima di tutto e in maniera violenta e dissacratoria a una cultura attardata, ritenuta "passatista" e propugna uno svecchiamento totale anche nella vita sociale e politica. La società italiana, soprattutto nell'Italia del nord, sta, infatti, evolvendo verso forme di economia industriale e le macchine hanno di fatto rivoluzionato il lavoro e la produzione, ma anche la vita. Il mito della macchina e del progresso è il primo ad essere inserito tra i valori futuristi, da esso derivano quello dell'automobile e quello della velocità.

Grazie all'abilità e al notevole attivismo del suo fondatore Marinetti, il futurismo ottiene un enorme successo tra gli uomini di cultura: la divulgazione delle tematiche futuriste ha luogo in incontri organizzati un po'ovunque, che spesso divengono anche serate di divertimento, come le famose "serate futuriste", nelle quali il pubblico è di proposito scandalizzato e messo in ridicolo da uno spirito anticonformista che anticipa atteggiamenti che dopo sarebbero stati sviluppati in Europa da altri movimenti d'avanguardia, come il dadaismo e in parte il surrealismo.

Di certo la determinazione ad appoggiare e promuovere tutto ciò che è nuovo e controcorrente spinge i futuristi a intraprendere sperimentazioni in tutti i campi della cultura, dalla letteratura alla poesia, alla grafica, alla pittura, alla scultura, all'architettura, alla fotografia, alla musica, alla danza, al cinema, alla moda, alla cucina. In tutte queste discipline l'aspetto che maggiormente si intende evidenziare è quello proprio del "movimento" fisico,contrapposto quindi alla staticità. Al movimento è connessa la dimensione della 
durata; entrambi è possibile esprimerli attraverso la scomposizione o la simultaneità della visione nel tempo ma anche nello spazio. L'interesse dei futuristi è centrato sulla contemporaneità e mai sulla storia; il loro agire è nell'immediato, pertanto investe la sfera sociale quanto la politica, arrivando a volte anche ad atteggiamenti velleitari, come l'interventismo nel 1915. Indubbia è stata la vicinanza di molti artisti del futurismo al fascismo, se non altro per le posizioni di nazionalismo che sempre ha assunto e anche perché Marinetti stesso lo abbraccia fin dagli albori, divenendo poi anche parlamentare. Sicuramente, tuttavia, il futurismo non è stato né omogeneo né monocorde; piuttosto è stato un fenomeno in grado di assorbire e in parte risolvere molte delle esigenze della società e della cultura di quel primo decennio del secolo.

Alcuni principi, alcune definizioni e alcuni tòpoi sono le chiavi di lettura di questo fenomeno culturale. In primo luogo i futuristi delineano il nemico da combattere, ossia il passatismo. Con questo neologismo, coniato nel 1910 nel manifesto Contro Venezia passatista dell'aprile, Marinetti, Boccioni, Carrà e Russolo mettono in chiaro che cosa e chi vogliono travolgere con le loro azioni; ma già sul manifesto scritto l'11 febbraio del 1909 e pubblicato poi il 20 dello stesso mese a Parigi si erano scagliati contro musei, biblioteche, accademie, contro tutto ciò che era tradizione e staticità in nome di nuove azioni e nuove creazioni.

L'automobile diviene, proprio grazie al futurismo, l'immagine simbolo del progresso già dagli albori del secolo. Molte delle opere di Balla sono ispirate all'automobile e al suo movimento. All'ebbrezza del dinamismo di un'auto in corsa si riferisce più volte Marinetti nel Manifesto fondante del movimento. La velocità del mezzo meccanico viene assimilata a ogni manifestazione dello stile di vita futurista, alle azioni stesse del vivere; è la stessa percezione della realtà a mutare seguendo, o cercando di seguire, il progresso tecno- 
logico. In ciò la filosofia che lo informa è confortata anche dalle teorie bergsoniane del continuo fluire. Ma per i futuristi il fluire è velocità e quindi sintesi di immagini moltiplicate e accavallate, che tengono conto non solo dell'oggetto ma anche dello spazio che lo circonda e del tempo nel quale il suo movimento viene agito. Vengono pertanto individuati, da Balla come da Russolo degli elementi formali in grado di rendere l'energia del moto, le sue linee-forza: i vettori. Proprio nel Manifesto del 1909 Marinetti dichiara: "la magnificenza del mondo si è arricchita di una bellezza nuova: la bellezza della velocità".

In sostanza il principio informatore del movimento, in ogni campo d'applicazione, è il dinamismo. Questo concetto è ben presente nel Manifesto tecnico della letteratura futurista del 1912, che tra l'altro incita a usare il verbo sempre all'infinito "per dare il senso della continuità della vita ed elasticità dell'intuizione che la percepisce". Sentire l'energia degli oggetti, la loro forza interna è l'esigenza primaria degli artisti futuristi, indicata anche nel Manifesto della scultura futurista firmato da Boccioni nel 1913, che invita a voler andare oltre la staticità cubista e a rendere la vita dinamica degli oggetti, reiterando nelle opere il gesto futurista, "la sensazione dinamica eternata come tale", come è scritto in Pittura futurista del 1910.

L'aeropittura costituisce l'ultima tematica connessa al movimento nel campo delle arti figurative dagli anni venti. Ad essa,ovviamente,si associa anche una "aeroplastica" relativa alla produzione tridimensionale e, soprattutto, ambientale. Nell'esigenza di rinnovare l'iconografia di un immaginario collettivo in evoluzione, l'attenzione dei futuristi si sposta sull'aereo, più moderno mezzo di trasporto, in grado di modificare con la sua veduta dall'alto e il suo viaggiare nell'atmosfera non solo i paesaggi ma anche le emozioni dei piloti (spesso gli stessi artisti) fino a restituire, oltre che visioni sintetiche, stati d'animo e sogni aerocosmici. Il primo 
artista a rappresentare il volo è Fedele Azari nel 1926 con Prospettive di volo, opera esposta alla Biennale di Venezia di quell'anno. Ma nel 1914 Marinetti aveva dato alle stampe L'aeroplano del papa. Romanzo profetico in versi liberi. Nel 1929 Marinetti insieme a Mino Somenzi redigono il Manifesto dell'Aeropittura, poi sottoscritto anche da altri. Nel $1931 \mathrm{gli}$ aerofuturisti espongono alla Galleria di Lino Pesaro a Milano e già nel catalogo manifestano eterogenei modi di intendere 1'“aeroarte". Per alcuni si trattava di un'evoluzione dell'“arte meccanica" di Balla, Depero e del primo Crali, mentre per altri e soprattutto dalla fine degli anni venti e per tutti i trenta si tratta di rappresentare sostanzialmente una visione o cosmica, come per Prampolini soprattutto ma anche per Oriani e Fillia, oppure fantasiosa, come per Dottori, Benedetta, Di Bosso, Delle Site, Peruzzi. Una visione di documentazione descrittiva dall'alto, artisticamente restituita, è presente in Tato, nel secondo Crali, in Ambrosi.

Altro elemento distintivo del futurismo, da principio in campo letterario, sono le parole in libertà,la cui teoria viene delineata e perfezionata da Marinetti prima di tutto nel Manifesto tecnico della letteratura futurista del 1912, in Risposte alle obiezioni di qualche mese dopo, in Distruzione della sintassi - Immaginazione senza fili - Parole in libertà dell'11 maggio 1913 e in Lo splendore geometrico e meccanico e la sensibilità numerica del 18 marzo 1914. In questo stesso periodo Marinetti dà alle stampe il poema Zang Tumb Tumb, reportage sull'assedio di Adrianopoli. Le "parole in libertà" rappresentano una vera e propria offensiva contro la linguistica italiana, quella poetica in primis, condotta con: la "distruzione della sintassi", 1" "abolizione della punteggiatura", 1'“immaginazione senza fili", l'“onomatopea", il "verbo all'infinito", l'introduzione in letteratura del rumore, del peso e dell'odore degli oggetti, la realizzazione del "brutto" e quant'altro. Ne consegue l'invenzione di una scrittura completamente rinnovata nella forma e nel significato, asso- 
lutamente non referenziale e altamente comunicativa. Nel 1914 Marinetti assorbe in letteratura e nel paroliberismo il concetto di simultaneità espresso già nel 1912 da pittori e scultori, che determina anche la nascita della poetica degli "stati d'animo" e l'obiettivo di giungere a una "multiforme prospettiva emozionale" e ciò porta anche a un'autentica svolta nel campo della grafica, che si arricchisce di nuovi elementi comunicativi, come il collage tipografico, anticipato sempre da Marinetti nel libro Les mots en liberté futuristes del 1919, sorta di summa dell'esperienza della teoria delle parole in libertà. Accanto a Marinetti si cimentano in questo campo Carrà e Soffici ma anche Depero nei poemi realizzati in "onomalingua" del 1915, Bruno Corra, Mario Crali e altri esponenti del secondo futurismo, soprattutto fiorentino; esso fu, altresì, fertile terreno per i dadaisti e i surrealisti come Breton e Tzara.

Lo spirito del futurismo è uno spirito d'avventura che, tuttavia, intende ancorarsi a un evidente pragmatismo fondato su un programma ben preciso; e il programma è espresso sempre nel manifesto, formula letteraria tra le più felici delle invenzioni del futurismo, capace di comunicare con forza e determinazione le nuove idee e di incidere profondamente in tutti i settori culturali. Ad ogni nuova impresa dei futuristi corrisponde un "manifesto" e ogni settore dell'arte, dalla letteratura alla pittura, alla musica, all'architettura, alla danza, alla fotografia, alla cinematografia, al teatro, alla moda, alla cucina ha il suo programma scritto di intenti, perentorio e battagliero. Tutti vengono redatti o tenuti a battesimo da Marinetti.

In tutti i manifesti futuristi il principio informatore è quello dell'azione. L'azione è sempre provocatoria, scandalistica e spavalda, che si attui in una poesia, in un dipinto, in una pellicola cinematografica, in un abito. Gli stessi titoli delle opere non celano questa carica di arroganza: provocando scandalo e divertimento, Palazzeschi scrive L'incendiario nel 1910, Govoni compone le Poesie elettriche del 1911, Carrà 
scrive con parole in libertà Guerrapittura nel 1915, Marinetti stesso dà alle stampe Distruzione nel 1920.

Anche le "serate futuriste" manifestano lo spirito irriverente e dissacratorio del movimento, teso a determinare con i propri comportamenti anticonformisti e beffardi reazioni altrettanto scomposte e fuori dalle regole. Vi volano improvvisamente pugni e schiaffi come abbracci, scoppiano petardi, insulti e quant'altro, inframmezzati da declamazioni poetiche e da musiche rumoristiche, a volte da esposizioni di pittura e scultura. Si tratta di sperimentazioni dell'impiego in simultaneità di più tipologie di "azioni" innovatrici che confluiranno teoricamente nella redazione del manifesto Ricostruzione futurista dell'universo, di Balla e Depero, pubblicato nel 1915; manifesto programmatico per uno stile di vita totalmente futurista.

Fino a tutti gli anni trenta questo titolo può essere applicabile a ogni intervento creativo futurista di tipo ambientale, scenico, allestitivo, cinematografico. Il futurismo vuole permeare di sé tutta la realtà oggettiva e comportamentale della società a esso contemporanea; dai "complessi plastici" di allestimento espositivo o scenografico alle "tavole tattili" all'innovazione della grafica, sia in letteratura sia in campo pubblicitario, la tensione creativa futurista trova sempre possibilità d'applicazione.

Non solo, i futuristi tendono alla "sinestesia", ossia a un'opera d'arte totale che implichi anche una partecipazione sensoriale a più livelli. Dai manifesti della musica del 1910 e dei drammaturghi si passa nel 1913 a quello della Pittura dei suoni, rumori e odori di Carrà e al prampoliniano $\mathrm{La}$ cromofonia e il valore degli spostamenti atmosferici e poi nel 1914 a Lo splendore geometrico e meccanico e la sensibilità numerica di Marinetti, in cui vengono presentate le "tavole sinottiche di valori lirici". La comunicazione futurista è composta in sostanza di interferenze sensoriali che mettono insieme il livello della visualità con quelli della verbalità e della gestualità. Balla e Depero raggiungono anche una maggiore 
consapevolezza della possibilità di realizzare il concetto di sinestesia nella costruzione del "complesso plastico". Depero aveva elaborato una prima stesura del manifesto già nel 1914, intitolata Complessità plastica - Gioco libero futurista - L'essere vivente artificiale; in essa la sinergia di poesia, pittura, scultura, musica genera una "plasticità complessa rumoristica - pittorica - psichica". Da questo momento in poi ogni altro manifesto settoriale terrà conto delle potenzialità espressive implicite nel coinvolgimento degli altri campi di interesse. Ma anche nell'ambito dell'arredo Balla e Depero, con le loro Case d'Arte Futurista a Roma e a Rovereto, come poi Prampolini sempre a Roma con la Casa d'Arte Italiana e altri in diverse città sparse per la penisola, programmano e progettano un nuovo tipo di arte decorativa futurista che sarà presentata a Parigi all'Esposizione Internazionale di Arti Decorative e Industriali del 1925 e che sarà pubblicizzata sulla rivista Noi di Prampolini.

La pubblicità, ossia la comunicazione a largo raggio d'azione è, tra l'altro, parte integrante del messaggio futurista. Essa è insita nella stessa necessità che il movimento ha fin dal suo nascere di divulgare e propagandare il rinnovamento culturale. Volantini, cartelloni, avvisi sui giornali, opuscoli spediti come "omaggio" non sono che il completamento dei manifesti programmatici che segnano le tappe e i traguardi della creatività sinestesica futurista. In questo Marinetti molto prende dalla pubblicità delle fabbriche e delle industrie, veicolando i suoi messaggi con mezzi fino a quel momento tipici della pubblicità di prodotti industriali e di uso quotidiano, che arrivano alla gente in maniera diretta, esplicita, come le Pillole Pink, il Cerotto Bertelli eccetera. D'altra parte è proprio Marinetti a inviare per posta il panettone come omaggio natalizio della sua rivista Poesia. Boccioni stesso, prima di aderire al futurismo, si cimenta nel campo della pubblicità così come tanti altri futuristi utilizzano nelle loro opere parolibere stralci di pubblicità. 
Sintesi dell'importanza dell'annuncio pubblicitario come veicolo d'informazione diretta e come modello di suggestione creativa è data da una celebre poesia di Aldo Palazzeschi intitolata "La passeggiata", il cui contenuto poetico è assunto dall'elencazione delle insegne di negozi e degli avvisi letti durante un percorso a piedi in una strada di città. La pubblicità futurista va d'altro canto a braccetto con la rivoluzione tipografica realizzata attraverso il "paroliberismo", che porta a un nuovo modo di concepire l'impaginazione e alla creazione del "lettering", ossia dei caratteri pubblicitari. Zang Tumb Tumb del 1914 di Marinetti esemplifica questo felice momento di sperimentazione. Altrettanto può sostenersi per le "lettere e i "numeri" animati di Cangiullo e per i "numeri architettonici" di Balla. La proliferazione delle riviste poi dà la possibilità di sperimentare una infinità di combinazioni grafiche fino a giungere ai fotomontaggi di Paladini e di Pannaggi. Per tutti gli anni venti e trenta esse rappresentano la possibilità di continuo approfondimento in questo specifico settore, considerato sempre di più un ambito di intervento futurista per eccellenza.

Nel 1931, a coronamento di una intesa speciale tra committente e creativo pubblicitario, Depero realizza il Numero unico futurista Campari, nel quale pubblica il manifesto Il futurismo e l'arte pubblicitaria, che costituisce un punto di arrivo importante nel processo di inserimento dell'arte nel circuito dell'industria e della produzione in genere e nella conseguente rivalutazione dell'arte cosiddetta "applicata". Nel 1928, a Torino, Prampolini era riuscito ad allestire un padiglione futurista all'interno del Parco del Valentino utilizzato per i festeggiamenti del decennale della vittoria. Nel padiglione, già di per sé un esempio di architettura effimera originale di tipo futurista, viene presentato il "cartello lanciatore", un modo di fare pubblicità che per dimensioni, per caratteri tipografici, per capacità comunicativa rappresenta il massimo raggiungimento dell'epoca. 